This article was downloaded by: [Staffordshire University]

On: 03 October 2014, At: 03:21

Publisher: Routledge

Informa Ltd Registered in England and Wales Registered Number: 1072954

Registered office: Mortimer House, 37-41 Mortimer Street, London W1T

3J H, UK

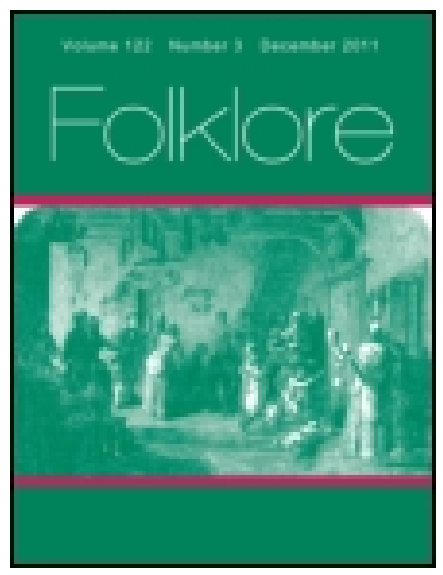

\title{
Folklore
}

Publication details, including instructions for authors and subscription information:

http:// www. tandfonline.com/loi/ rfol20

\section{Legends of the Cars}

M. C. Balfour

Published online: 14 Feb 2012.

To cite this article: M. C. Balfour (1891) Legends of the Cars, Folklore, 2:2, 145-170, DOI: $10.1080 / 0015587 X .1891 .9720054$

To link to this article: http:// dx. doi. org/ 10.1080/0015587X.1891.9720054

\section{PLEASE SCROLL DOWN FOR ARTICLE}

Taylor \& Francis makes every effort to ensure the accuracy of all the information (the "Content") contained in the publications on our platform. However, Taylor \& Francis, our agents, and our licensors make no representations or warranties whatsoever as to the accuracy, completeness, or suitability for any purpose of the Content. Any opinions and views expressed in this publication are the opinions and views of the authors, and are not the views of or endorsed by Taylor \& Francis. The accuracy of the Content should not be relied upon and should be independently verified with primary sources of information. Taylor and Francis shall not be liable for any losses, actions, claims, proceedings, demands, costs, expenses, damages, and other liabilities whatsoever or howsoever caused arising directly or indirectly in connection with, in relation to or arising out of the use of the Content.

This article may be used for research, teaching, and private study purposes. Any substantial or systematic reproduction, redistribution, reselling, loan, sub-licensing, systematic supply, or distribution in any form to anyone is 
expressly forbidden. Terms $\&$ Conditions of access and use can be found at http://www.tandfonline.com/page/terms-and-conditions 


\section{Jfolk= ilore.}

VOL II.] JUNE, 1891. [No. II.

\section{LEGENDS OF THE CARS.}

T FANCY that many people still picture Lincolnshire 1 to themselves as a region of bogs and swamps, of fever-haunted marshes, and ague-infested lowlands. I know that I, personally, expected something of the sort, when I first entered the county, and in speaking about it to strangers, their first remark is apt to be, that we must have suffered much in those "dreadful fens". Now this is an entirely mistaken idea of the shire.

Even in the South, the true fen district, the drainage system has been so widely carried out, that I am told the great marshes have been almost entirely reclaimed, and many hundreds of useless acres are now turned into fertile farm-lands. If this be true of the South, it is much more so of the Northern Division, which, to begin with, has in general a higher average level, and is more uneven in its surface, being also traversed by two long low hill ranges from N.IV. to S.E. In the parts of Lindsey, there are no fens, their place being taken by the Cars, which were once wide swamps, bordering the course of a small stream or river. These have been drained, and I do not think that any now exist in their old barren condition, so great is the change that has taken place during the last half century: Broad dykes now intersect the fertile fields, and run beside the roads, on their way to join a central canal which vol. 11.

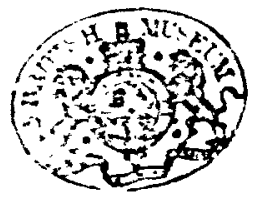


carries the waters of the district to the sea, the original river meandering now on one side now on the other, a mere brook of but a few feet wide, often dried up in summer. Drained cars like these lie along the wide shallow valley of the Ancholme, between the parallel ranges of the Wolds and the Cliffs ; the original Ancholme, a tiny twisted stream, being replaced, both in name and use, by a broad canal, which runs northwards for some thirty miles, as straight as an arrow, to join at last the wide Estuary of the Humber.

Were this the place, I might speak of the elaborate system for regulating the outlet of the water; of the yawning dykes that border or cross the roads, making them by no means safe on dark nights, holding, as they do, from two to ten feet of water and many more of shiny treacherous mud; or of the lonely dwellings along the Ancholme banks, only to be reached by a narrow bridlepath, with bewildering lancs of water on either hand, where a horse must be blindfolded before it will cross the frail wooden bridges over the noisy water gates at the joining of the dykes with the main Canal; but I am more concerned at present with memories of the Cars as they once were, a wild desolate dreary marsh, full of strange sights and sounds, than as they now exist.

Nevertheless they are still worth seeing, and have a beauty, or rather an attraction of their own. Stunted willows mark the dyke-sides, and in winter there are wide stretches of black glistening peat-lands and damp pastures; here and there great black snags work their way up from submerged forests below. When the mists rise at dusk in shifting wreaths, and the bleak wind from the North Sea moans and whistles across the valley, it is not difficult to people the Cars once more with all the uncanny dwellers, whose memory is preserved in the old stories. Then in summer, with its charm of wide vision, and something of the amplitude and serenity of the sea, in its stretching levels and far-off horizon, it scems to hold the brightness 
and reflect the gloom of the great arch of heaven overhead, in plots of vivid greenery and waving corn, and a maze of glittering dancing lanes of water. At all times, it seems a fit resting-place for the last days of a dying mythology.

With the barren Cars of the older times is connected a peasantry that is changing as the soil itself has changed, only more gradually, for the sluggish current of their life and habit is but slowly beaten back by the impetus of modern innovations. Still, in time, the running water carries awvay the stagnant, and so already, it is only here and there that one can find traces of the poor ague-shaken, opium-eating creatures of earlier times. Many an old woman eats opium openly, and I fear all the men who can get it-will drink gin. But the days are gone by when the one or the other was in constant and daily need, to still the shaking or deaden the misery born of the fevermists and stagnant pools. Nevertheless, whether it be due to the climate, or the scarcity of railways, or the character of the people themselves, civilization scems a long way behindhand in North Lincolnshire, when compared with other parts of England.

It secms as if it were off the high-road, so to speak, of busy modern English life. Lindsey is entirely agricultural, and in these days of depression amongst farmers, and of absentee landlords, it is visited by few strangers ; and the only resident upper class is represented by the clergy, and a very mixed set of tenant-farmers, who, in trouble themselves, generally care little for the people under them, except as regards their work and pay.

This is, I dare say, unavoidable; but it throws the people back on themselves, and accounts, no doubt, for the survival of much amongst them which has decayed elsewhere. Even their speech sounds strange to a modern English ear, for it is almost pure Saxon, and keeps many of the original inflexions which we have lost. Certainly. it bears signs of the many races that have dwelt in Lincolnshire, and surely no county in England has known more 
varied masters ; there are many Norse and Danish words, and some Roman and Norman names; but in the common speech, French and Latin derivatives are conspicuous by their absence.

The people themselves are not easy to make friends with, for they are strongly suspicious of strangers; but once won over, are said to be staunch and faithful. They are grave, long-featured, and rather melancholy in face, touchy and reserved in disposition, and intensely averse to change or innovation of any sort; many of them live and die within the limits of a narrow parish, outside of which they never set foot. The younger generations are changing; but they show less disbelief in the old legends than indifference to them; they seem growing, not so much less superstitious, as less impressionable. But in some of the old people, there is still a simple serious faith that is delightful, and I do not think that elsewhere in England one could nowadays find such a childlike certainty of unseen things or such an unquestioning belief in supernatural powers.

I have given this slight outline of the district and some of its inhabitants, in order to show amid what surroundings linger these wild tales of witchcraft, and the spiritworld, in this little isolated home of folk-lore. Here, in this bleak and lonely tract, scarcely yet won over to civilization, has dwelt for ages a people, ignorant, povertystricken, weakened by malaria, and strongly affected by their wild home; and here still, amongst a few elders, who remember the traditions of their youth, and the beliefs of their fathers, linger tales that tell of the old pagan customs, that have perhaps existed in these parts since the very dawn of history.

I have gathered together a number of these storiessome of them were told me by devout believers, mostly aged folk, who dated from the days of universal credulity; some were repeated as "my grandad used to tell"-by younger people, and some were pieced together by scraps 


\section{Legends of the Cars.}

gained from several sources. One, which I call "The Dead Moon", I first heard of in a sort of nursery rhyme some children were singing. I have listened to awesome tales of "boggarts" and "todlowries" that have still local habitations as well as names; and to weird stories of witches, and woe-women and their spells, till I nearly believed in them myself; and to strange, rambling histories that seemed like peeps into a bygone world, where the fantastic spirits were more real than the trembling, fearing, conciliating people they alternately helped and oppressed. I fear I cannot preserve the rude poetry and grace of the vernacular; but I tell these stories of the Cars of the Ancholme Valley exactly as told to me, lest in altering I might spoil them. I heard the first from an aged woman, a life-long dweller in these Cars, who in her young days herself observed the rite she describes, though she would not confess to it within the hearing of her grand-children, whose indifference and disbelief shocked her greatly. To her, "Tiddy MIun" was a perfect reality, and one to be loved as well as feared. She is now dead, and I doubt whether anyone else knows the legend, which she said had been forgotten for many, many years, by all but herself and one or two old friends, all gone before her.

I think the legend is, if not so poetical as some, at least a curious one; and particularly so, as showing the innately heathen idea of propitiation by offerings. There is an inconsequence and an incompleteness about it for which I am not responsible; I tell it as it was told to me, and I have tried to keep to the old woman's words as closely as possible, only changing them where they would certainly not be "understanded of the people" without an intimate knowledge of the dialect.

\section{"TIDDY MUN."}

Whiles syne, afore tha dykes wor made, an' tha river-bed changed, whan tha Cars wor nobbut bog-lands, an' full o' watter-holes ; tha wor teemin, as thou mayst a' heerd " $1 i^{\prime}$ 
Boggarts and Will-o'-tha-IVykes, an' sich loike ; voices o' deed folks, an' hands wi'outen airms, that came i' tha darklins, moanin' an' cryin an' beckonin' all night thruff; todlowries dancin' on tha tussocks, an' witches ridin' on tha great black snags, that turned to snakes, an' raced about wi' 'em i' tha watter; my word! 'twor a stra-ange an' ill place to be in, come evens.

Folk wor gey skeered on un nat'rally, an' wouldna goo nigh un wi'outen a charm o' some sort, just a witches pink or a Bible-ball, or the loike o' that. A'll tell thee 'bout them another toime. Tha shook wi' froight, a tell thee, whan tha found their sels, i' tha Cars at darklins. For sartain, tha wor mostly shakin 'i' they toimes ; for tha agur an' fever were terrible bad, an' thar wor poor weak crysoms, fit for nowt but to soop gin an' eat op'um. In ma young days, we'd all tha agur; tha women ower tha fire, tha men out i' tha garth, even tha bairns had tha shakes reg'lar. Ay mebbe, tha's better off noo, but a don't know, a don't know, tha's lost Tiddy Mun. Weel, weel. Tha kenned foine that tha fever an' agur comed fra tha bogs, but so come as tha heerd tell, that tha ma-ashes mun be drained as tha ca' it, tha wor sore miscontented, for tha wor used to un, an' ther feythers afore em', an' tha thowt, as tha sayin' is, bad's bad, but meddlins wuss.

Tha tell't un fine tales, 'at tha mists 'ud lift, an' tha bogs 'ud come i' tha molds, an' th'ud be no'on agur; but tha misliked tha changement, an' wor main fratched wi' tha Dutchies, who comed across tha seas for tha delvin.

Tha folk would na give tha Duchies vittles, or beddin', or fair words; no'on let 'em cross tha door-sill; an' tha said to each ither, tha said, as t'ud be ill days for the Cars, an' tha poor Car-folk, so-be tha bog-holes wor meddled wi', an' "Tiddy Mun" wor unhapped.

For thee know'st, Tiddy Mun dwelt in tha watter-holes doun deep i' tha green still watter, an' a comed out nobbut of evens, whan tha mists rose. Than a comed crappelin out i'tha darklins, limpelty lobelty, like a dearie wee au'd 
gran'ther, wi' lang white hair, an' a lang white beardie, all cotted an' tangled together; limpelty-lobelty, an' a gowned i' gray, while tha could scarce see un thruff tha mist, an' a come wi' a sound o' rinnin' watter, an' a sough o' wind, an' laughin' like tha pyewipe screech. Tha wor none so skeered on Tiddy Mun like tha boggarts an' such hawiver. A worn't wicked an' tantrummy like tha watterwives; an' a worn't white an' creepy like tha Dead Hands. But natheless, 'twor sort o' shivery like when tha set round tha fire, to hear the screechin' laugh out by the door, passin' in a skirl o' wind an' watter; still tha only pulled in a bit nigher together, an' lispit wi' a keek ower tha shouther, "'Arken to Tiddy Mun!"

Mind ye, tha au'd Mun hurted none, nay, a wor real good to un at times. Whan tha year wor geyan wet, and tha watter rose $i$ ' tha marshes, while it creepit up to the docrsill, an' covered tha pads, come tha fust New Moon, tha feyther an' mither, an' a' tha brats, ud go out i' tha darklins, an' lookin' ower the bog, called out together, thoff mappen a bit skeered an' quavery' like :

"Tiddy Mun, wi'-out a name,
tha watters thruff!"

an' all holdin' on togither an' tremblin', a'd stan' shakin' an' shiverin', while tha heerd tha pyewipe screech 'cross tha swamp; 'twor tha au'd MIun's holla! an'i'tha mom, sure 'nough, tha watter ud be doun, an' tha pads dry. Tiddy Mun a done tha job for un.

What's that ? Ay' a called 'un Tiddy Mun, for a wor none bigger 'n a three year's bairn, but a hadn't rightly no sort of a name-a niver had nonc. Someday a'll tell thee how that comed.

So's a wor sayin'. Tiddy Mun dwelt i' tha watter-holes, an' noo tha Dutchies wor a emptyin' 'cm out, while a wor dry as a two year au'd Motherin cake-an' thou'll no take much o' that. Hast heard tha au'd rhyme, as says : 


\author{
"Tiddy Mun, wi-out a name \\ White heed, walkin' lame; \\ While tha watter teems tha fen \\ Tiddy Mun 'll harm nane."
}

An' this wor tha pother! for tha watter-holes wor most dry, an' tha watter wor drawd off into big dykes, so that tha soppy, quiverin' bog wor turnin' in firm molds, an' wheer'd Tiddy Mun be than? Iverybody said, as ill times wor comin' for tha Cars.

But, however, tha wor no help for 't; tha Dutchies delved, an' tha' Dutchies drawd tha watter off, an' tha dykes gotten ever langer an' langer, an' deeper an' deeper ; tha watter runned away, an' runned away down to tha river, an' tha black soft bog-lands 'ud soon be turned to green closin's.

But thoff tha work gotten done, it wor no'on wi' out trouble. At the Inn o' nights, on tha great settle, an' $i$ ' tha garths, an' $i$ ' tha kitchens to home, tha lispit strange an' queer tales, ay dearie me, stra'ange an' queer, but 'true's death ! an' tha au'd folk wagged ther heads, an' tha young uns wagged ther tongues, an' tha anes thowt, an' tha ithers said :

"Ay, an' for sure, it's ill comes o' crossin' Tiddy Mun!" For mark ma words! 'twar first ane, syne anither o'tha Dutchies wor gone, clean sperrited away! not a sight o'un anywheres! tha sowt for un, an' sowt for un, but no'on a shadow of un wor iver seen more, an' tha Car-folk kenned fine, that a'd niver find un, nay, not if a sowt till tha gowden Beasts o' Judgement come a-roarin' an' a rampin' ower tha land, for to fett tha sinners.

Tiddy Mun a'd fetted un away, an' drooned un i' tha mud holes, wheer tha hadn't drawed off all tha watter!

An' tha Car-folk nodded an' said :

"Ay, that comed o' crossin' Tiddy MIun!"

But tha browt more Dutchies for tha work, an' thoff Tiddy Mun fetted un, an' fetted un, tha work gotten on natheless an' tha wor no help for 't. 
$A n^{\prime}$ soon tha poor Car-folk kennt that tha au'd Mun wor sore fratched wi iverybody.

For soon a sneepit all $i$ ' turn: tha coos pined, tha pigs starved, an' tha pownies went lame; tha brats took sick, tha lambs dwined, tha creed meal brunt 'issen, an' tha new milk craddled ; tha thatch fell in, an' tha walls burst out, an' all an' anders went arsy'varsy:

At first tha Car-folk couldna think 'at tha au'd Mun 'ud worritt's ain people sich an' away; an' a thought mayhap 'twor tha witches or tha tod-lowries, as done it. So tha lads stoned tha wall-eyed witch up to Gorby out o' tha Market-Place, an' Sally to Wadham wi' tha Evil Eye, she as charmed the dead men out o' ther graves, i' tha kirk garths; tha ducked she in tha horse-pond while a wor most dead; an' tha all said "our father" back'ards an' spat to the east to keep tha tod-lowries' pranks of ; but 'twor no'on helping; for Tiddy Mun 'isself wor angered, an' a wor visitin' it on 's poor Car-folks. An' what could tha do?

The bairns sickened i' ther mothers' airms ; an' ther poor white faces niver brightened oop ; an' tha feythers sat an' smoked, while tha mothers grat, ower tha tiddy innocent babbies lyin' theer so white an' smilin' an' peaceful. 'Twor like a frost 'at comes an' kills the bonniest flowers. But tha hearts wor sore, an' ther stomachs empty, wi' all this sickness an' bad harvest an' what not; an' somethin' mun be done, or the Car-folk 'ud soon be a' deed an' gone.

Endlins, some 'un minded how, whan tha watters rose i' tha marshes, afore tha delvin'; an' tha folk ca'ed out to Tiddy Mun, come New Moon i' tha darklins; a heerd un an' did as a wor axed. An' tha thowt, mappen if tha ca'd un age'an, so's to show un like, as tha Car-folk wished un well, an' that a'd give un tha watter back if tha only could-maybe a'd take tha bad spell undone, and forgive 'un again.

So tha fixed 'at tha should a' meet togither come tha next New Moon doun by tha cross dyke, ly tha au'd stope nigh on to John Ratton's garth. 
Weel, 'twor a reg'lar gath'rin', there wor au'd Tom o' tha Hatch an' IVillem, his sister's son, from Priestrigg; an' crooked Fred Lidgitt, an' Brock o' Hell-gate, an' Ted Badley, as wor feyther's brothers to me; an' lots more on 'em, wi' women-folk an' bairns. A'll no say a warna theer masel, just mappen, thee knawst!

Tha comed i' threes an' fowers, joompin' at ivery sough $o$ ' wind, an' screechin' at ivery snag, but tha didn't need, for tha poor au'd Boggarts an' Jack o' Lanterns wor clean delved away. Mebbe ther's boggarts an' bogles still, an' witches an' things, a dunnot say; but they good au'd times is gone $i$ ' tha marshes, an' tha poor swamp-bogles mun flit wi' tha watter an' a seen 'em go, mysel.

But, hawiver, as a wor sayin, tha comed, every one wi' a stoup o' fresh watler in 's hand; an' whiles it darkened, tha stood a' togithur, lispin' an' flusterin', keekin' i' tha shades ower tha shouthers, an' 'arkenin' oneasy-like to tha skirlin' o' tha wind, an' tha lip-lap o' tha rinnin' watter.

Come tha darklins at long last, an' tha stood all on 'em at tha dyke-edge, an' lookin ower to tha new River, tha ca'd out a' togither, stra'ange an' loud,

"Tiddy Mun, wi-out a name,

Here's watter for thee, tak' tha spell undone!"

an' tha teemed tha watter out $o^{\prime}$ tha stoups in tha dyke splash sploppert!

'Twor geyan skeerful, stannin' holdin' on togither, $i$ tha stillness. Tha 'arkened wi' all ther might, to hear if Tiddy Mun answered 'em; but ther wor nothing but on-natral stillness. An' then, just whan tha thowt 'twor no'on good, ther broke out tha awfullest wailin' an' whimperin' all round about 'em; it comed back'ards an' for'ards, for all tha world like a lot o' little cryin' babbies greetin' as if to break ther hearts, an' none to comfort 'em : a sobbed an' sobbed thersels most quiet, an' then began again louder 'n ever, wailin' an moanin' till a made uns heart ache to hear 'em. 
An' all to wanst the mothers cried out as 'twor ther dead bairns, callin' on Tiddy Mun to tak tha spell undone, an' let tha childer live an' grow strong ; an' tha pore innocents, fleein' above us i' tha darklins, moaned an' whimpered soft-like, as if thea kenned ther mothers'voices an' wor tryin' to reach ther bosom. An' tha wor women as said 'at tiddy hands 'ad touched 'em, an' cold lips kissed 'em, an' soft wings fluttered round 'em that night, as tha stood waitin' an' arkenin' to tha woful greetin'. Then all at once, tha wor stillness agean, an' tha could hear tha watter lappin' at ther feet, an' tha dog ye'ppin' $i$ ' tha garth. But then comed soft an' fond-like from tha river hissen, th' aud pyewipe screech, once an' again a comed, an' fortrue, 'twor tha aud man's holler. An' tha kenned a'd tak tha spell undone, for 'twor so kind an' broodlin' an' sorry-like as never was.

Ay dearie day! how tha laughed an' grat together, runnin' an' jumpin' about, like a pack o' brats comin' out o' school, as tha set off home, wi' light hearts, an' never a thought on tha boggarts. Only tha mothers thought o' ther dead babies an' ther arms felt empty an' ther hearts lonesome an' wearyin' for tha cold kiss an' tha flutterin' $o^{\prime}$ tha tiddy fingers, an' tha grat wi' thinkin' on ther poor wee bodies, driftin' aboot i' tha soughin' o' tha night win'.

But fro' that day, mark ma words! 'twor strange an' thrivin' $i$ ' tha Cars. Tha sick bairns gotten well, an' tha cattle throve, an' tha bacon-pigs fattened; tha men folk addled good wages, an' bread tror plenty ; fur Tiddy Mun had taken tha bad spell undone. But every New Mloon as was, out tha went in tha darklins, to tha gainest dykeedge, feyther an' mither an' brats; an' tha tecmed tha watter i' tha dyke cryin',

"Tiddy Mun wi-out a name

Here's watter for thee :"

An' tha pyewipe screech 'ud come back, soft and tender an' pleased. But for certain-sure, if wan o' un didna go out. 
c'ep a wor sick, Tiddy MIun missed un, an wor angered wi' un, an' laid tha spell on 'un 'arder nor ever ; while a went wi' tha others, come next New MIoon, to ax tha spell undone. An' whan tha bairns wor bad, a tellt un as Tiddy Mun 'ud fett 'em away; an' a wor good as gold to once, for tha kenned as a'd do it.

But thae days is gone by, an' folk now ken nowt about un. Ay, faix, is it true for a' that; a've seen un mysel, limpin' by i' tha fog, all grey an' white an' screechin' like tha pyewipe, but 'tis lang syne a's ben by, an' a've teemed tha watter out $o$ ' tha stoup too, but a'm too aud now, thou seest, an' a cannot walk, since years gone. But a guess Tiddy Mun's bin' frighted away wi' a' tha new ways an' gear, for folk dinna ken un no more, an' a niver hear say now, as we used to say when a wor young, an' annybody had a mort o'trouble an' mischance, an' wry luck, us said,

"Ah, thou arnt bin out $i$ ' tha New Moon lately, an' for certain-sure, it's ill to cross Tiddy Mun wi-out a name!"

The next legend was obtained from a young girl of nine, a cripple, who stated that she had heard it from her "gran." But I think it was tinged by her own fancy, which seemed to lean to eerie things, and she certainly revelled in the gruesome descriptions, fairly making my flesh creep with her words and gestures. I have kept not only to the outline of her story, but in great part to her very words, which I think I could not have made more effective even if I had wished to do so. 


\section{TIIE DEAD Moon.}

Long agone, i'ma gran's toime, th' Car-lan's doun by wor $a^{\prime}$ in bogs, as thee's heerd tell, mebbe : gra'at pools o' black watter, an' creepin' trickles o' green watter, an' squishy mools as'd soock owt in, as stept on un.

Weel, my gran' used to sa'ay, how, long afwore her toime, tha moon's sel' wor towanst de'ad an' buried i' tha ma-ashes; an' if thee will, a'll tell thee aboot it as she used for to tell me.

Tha moon up yond', shone an' shone to than, jest as she do now, thoff thou moightn't ha' thowt it; an' whan she shone, she loighted oop a' tha bog-pads so's a body cu'd wa'alk aboot, most 's safe as o'day's. But when she didna shine, then oot cam' a tha Things 'at dool i' tha Darkness, an' want aboot seekin' to do evil an' harm to all as worna safe beside ther ain he'arths. Harm an' mischance an' mischief : Bogles, an' de'ad Things, an' crawlin' Horrors: tha a' coomed oot o' noights when the moon didna shine.

Weel, it comed so, 'at tha Moon heerd tell on a' this; an' bein' kin' an' good-as she be, surely, a-shoinin' fur us a' noights, 'stead o' takin' her nat'ral rest; she wor main troubled to think o' what went on ahint her back, loike; an' says she: "A'll see fur mysel, a wull; it mebbe, 'at its none so bad 's fo'ak mak' oot."

So sewer 'nuff, come tha month end, doun she stept hapt oop wi' a black cloak, an' a black hood o'wer her yaller shinin' hair ; an' straight she went to tha Bog edge, an' looked aboot her. IVatter here, an' watter there ; wavin' tussocks, an' trem'lin' mools, an' gra'at black snags a' twisted and bent; an' afwore her, a' dark-dark, but the glimmer $o^{\prime}$ tha stars on tha pools, an' tha loight as comed fro' 's 'ain white feet, stcalin' oot o' s black clo'ak. On a went, fair into the mid' o' tha bogs; an' aye lookin' about 
her; an' 'twor a mortal quare soight as 'a looked on. Tha witches girned as tha rode past on ther gra'at black cats; an' tha evil Eye glowered fro' tha da'arkest comers-an' tha will-o'-tha-wykes danced a' aboot wi'ther lanterns swingin' o' ther backs. Than tha de'ad fo'ak rose i' tha watter, an' lookit roon' 'em in white twisted fa'aces an' hell fire i' ther empty een-holes ; an' tha slimy drippin' De'ad Han's slithered aboot, beckonin' an' p'intin', and makin' yer skin crawl wi' ther cowld wet feel.

Tha moon drew 's clo'ak faster aboot her, an' tremmelt; but a wouldna gaw back, wi'oot seein' a' ther wor to be seen, so on she went, steppin' light as tha win' in summer fro' tuft to tuft, atween tha greedy gurglin' watter ho'als; an' jest as she comed nigh a big black pool, 's fut slipt, and a wor nigh toomlin' in-an' a grabbed wi' bo'oth han's at a snag near by, to steady 'asel' wi' ; but so cum as she touched it, a twined itsel' round her wrists loike a pa'ir o' han'cuffs, an' gript her so 's she culdna move. She pulled, an' twisted, an' fowt, but twor no'on good : a wor fast, an' a mo'ost sta'ay fast ; so a' lookit aboot, an' wunnerd if help 'd coom by; but a saw nowt but shiftin' flurryin' evil Things, comin' an' goin' here an' there busy wi' ther ain ill wark.

But presently, as a stood trem'lin' $i$ ' tha da'ark a heerd summat ca'allin' $i$ ' tha distance-a voice 'at ca'alled an' ca'all'd, an' than de'ed away wi' a sob; an' then began agean wi' a screech o' pain or fear, an' ca'd an' ca'd, till tha ma-ashes weer full on tha pitiful cryin' voice; an' than a heerd steps floonderin' along, squishin' $i$ ' tha muck, an' slippin' on' tha tufts ; an' throff tha darkness, a saw han's catchin' at the snags an' tha tussocks, an' a white face wi' gre'at feared eyen.

'Twor a man strayed $i$ ' tha bogs ; an' a' roon' about un tha girnin' bogles, an' tha de'ad fo'ak, an' tha creepin' Horrors crawled an' crooded ; tha voices mocked un, an' the De'ad Han's ploocked at un, an' ahead, tha will o' tha wykes dangelt ther lanterns, an' shuk wi' evil glee as a led un furder 'n furder fro' tha reet track. Ma-azed wi' fear 
an' loathin' for tha Things aboot un, a stroogled on tords tha flick'rin' loights 'at looked loike he'p an' sa'afety.

"Thou yonder," a'd shriek, "Thou!-a'm catched i' tha bog-lan's!-dost hear?-God an' Mary save 's fro' the Horrors-he'p, thou yonder!' An' then a'd stop an' sob an' moan an' ca' on a' tha saints an' wise women an' God 'issel to fetch un oot.

An' than 'a 'd break oot in a shriek age'an, as tha slimy slithery things crawled round un, till a couldna even see the fause lights afwore un. An' than, 's if 'tworna bad aneugh a'ready, the horrors ' $d$ tak a' sorts o' shapes; an' rampin' lasses 'd keek at un wi' bright eyen, an' stretch oot soft he'pin' han's; but when a'd try to catch hol' on un, a'd cha'ange in 's grip to slimy things an' shapeless worms, an' tha wicked voices 'd mock un wi' foul glee. An a' tha evil thoughts an' deeds o's life cam' an' whispet in 's ears, an' da'anced aboot an' shooted oot tha secret things o's ain heart, till a shrieked an' sobbed wi' pain an' shame, an' the Horrors crawled an' gibbered roon' aboot an' mocked un. An' when tha poor Moon saw 'at he wor coomin' nigher an' nigher to the deep holes an' tha deadly quicks, an' furder 'n furder fro' the pad, a wor so mad an' so sorry, 'at she stroogled an' fowt an' pulled, harder nor iver. An' thoff a couldna get loose, wi' a' her twistin' an' toogin', the black hood fell ba'ack off 'a shoinin' yaller hair, an' tha beautiful light as coomed fro't druv away tha darkness.

Ooh! but tha man grat wi' joy to see God's ain light age'an; an' towanst tha evil things fled ba'ack into tha da'ark corners; fur tha canna boide tha light. So tha left un, an' fled ; an' a could see whur a wor, and whur tha pad wor, an' hoo a'd hev to gaw fur to get oot o' tha ma'ash. An' a wor in sich a ha-aste to get awa-ay fro' tha quicks an' tha boglan's, an' tha things 'at doolt thur, 'at a sca'arce lookit at tha bra'ave light 'at coomed fro' tha beautiful shinin' yaller hair streamin' oot o'er the black cloak, an' fallin' to the watter at's feet. An' tha Moon's sel wor so tuk oop wi' sa'avin' hc, an' wi' rejoicin' 'at a wor ba'ack on 
tha reet pad, 'at a cle'an furgot 'at a needed he'p 'asel', an' 'at a wor held fast by the Black Snag.

So off a went ; spent an' gaspin' an' stumblin', an' sobbin' wi' joy, fleein' fur's life oot o' tha tur'ble Bogs. Than it coom ower the Moon, 'at 'ad loike main to gaw wi' un ; an' a gra'at fear coom to 'a ; an' a pulled an' fowt 'sif a wor mad, till a fell on's knees, spent wi' toogin', at tha fut o' tha snag. An' as a la'ay thur, gaspin' fur bre'ath, tha bla'ack hood fell for'ard ower her he'ad; an' thoff she tried to throw un ba'ack, 'twor catched in her hair, an' wudna gaw. So oot want tha blessed light, an' back coomed tha darkness wi' a' its evil things, wi' a screech an' a howl. They cam croodin' round her, mockin' an' snatchin' an' beatin'; shriekin' wi' rage an' spite, an' swearin' wi' foul tongues, spittin' an' snarlin', fur tha kenned her fur ther au'd enemy, tha' bra'ave bright Moon, as druv 'em ba'ack into tha corners, an' kep'em fro' warkin' their wicked wills My-what a clapperdatch 'twor-an' tha poor Moon crooched tremblin' an' sick i'tha mid, an' won'erd when tha'd make an en' o't, an' o' she.

"Dom' tha !" yelled tha witch-bodies, "thou'st spiled oor spells this year agone!"

"An' thou keeps us in wer stra'ight coffins o'noights!" mo'aned tha de'id Fo'ak.

"An' us thou sen's to brood $i$ tha corners!" howled tha Bogles.

An' a' tha Things joined in wi' a gra'at " Ho, ho!" till tha varry tussocks shuk, and tha watter gurgled. An' tha began age'an.

"Us'll poison her-poison her !" shrieked the witches.

An' "Ho, ho!" howled the Things age'an.

"Us'll smother her-smother her!" whispered the crawlin' Horrors, an' twined thersel's roon' her knees.

An' "Ho, ho!" mocked the rest o'un.

"Us'll strangle her-strangle her!" screeched tha De'ad Han's, an' ploocked at 'a throat wi' could fingers.

An' "Ho, ho!" they yelled age'an. 
But tha dead Fo'ak writhed an' girned about 'a, an' chuckled to thersel's.

"We'se bury thee, bury thec, doun wi' us i' tha black mools!"

An' age'an tha a' shouted wi' spite an' ill-will. An' tha poor Moon crooched doun, an' wished a wor de'ad, an' done wi'.

An' tha fowt an' squabbled what tha should do wi' her, till a pale gray light began to coom i' tha sky; an' it drew nigh the dawning. $A n^{\prime}$ when tha saw that, tha wor feared lest tha shouldna hev toime to work ther wull; an' tha catched hol' on her, wi' horrid bony fingers, an' laid her deep i' tha watter at fut o' tha snag. An' tha dead fo'ak held her doun, while tha bogles fo't a stra'ange big sto'an an' rowled it o'top o' her, to keep her fro' rising. An' tha towld twae o' tha will o' tha wykes to ta'ake turns i' watchin', on tha black snag, to sec 'at a lay safe an' still, an' couldna get oot to spoil ther sport wi' her loight, nor to he'p tha poor car-fo'ak to keep oot o' tha quicks an' ho'als o'nights. An' then, as tha grey light comed brighter i' tha sky, tha shapeless Things fled away to seek tha da'ark corners, an' tha dead fo'ak crept ba'ack into tha watter, or crammed thersel's into ther coffins, and tha witches went ho'am to ther ill-do'ins. An' tha green slimy watter shone i' tha dawnin' 'sif nae ill thing 'd aye coom nigh it.

An' thur lay tha poor moon, de'ad an' buried i' tha bog till sum 'un 'd set her loose; an' who'd ken whur to look fur a?

Weel, tha days pa'assed, an' 'twor tha toime fur tha new moon's coomin'; an' tha fo'ak put pennies i' ther pockets, and straws $i$ ' ther caps so's to be ready fur a, an lookit aboot onquietly, fur tha moon wor a good frien' to tha ma'ash fo'ak, an' tha wor main glad when tha da'ark toime wor ga'an, an' tha pads wor safe age'an, an' tha Evil Things wor druv back by the blessed Light into the darkness an' tha watter ho'als. 
But days an' da'ays passed, an' tha new moon niver ca'ame, an' tha nights wor aye da'ark, an' th' Evil Things wor badder nor iver. Ther wor no 'on a loaning safe to travel, an' tha boggarts crept an' wailed roon' tha hooses an' keekit in at the winders, an' sneepit at tha latches, till tha poor bodies mun ke'p lights a' night, else tha horrors 'd a coomed ower tha varry doorsils.

Aye so, tha bogles o' a' sorts seemed to ha' lost a' fearin'. Tha howled an' lafft an' screecht aroon', fit to wa'ake tha de'id thersel's, an' tha Car-fo'ak mun sit tremmlin' an' shakin' by tha foire, an' could nor sleep nor rast, nor pit fit across tha sil', a' thae da'ark an' dreary nights.

An' still tha da'ays went on, an' tha new moon niver comed.

Nat'rally tha poor fo'ak were stra'ange feared and mazed, an' a lot $o$ ' un went to the wise woman wha doolt $i$ ' th' 'owd mill, an' axed ef so be 's tha could fin' oot wheer tha moon wor ga'an.

"Weel," said she, arter lookin' i' tha brewpot, and i' tha mirror, an' $i$ ' tha Book, "it be main quare, but a canna reetly tell ye what's hapt wi' her. It be dark, dark, an' a canna see nowt i' tha spells. Go'a slow, childer, a 'll think on it, an' mappen a 'll can he'p ye yet. If ye hear o' a'wthing, coom by 'n tell ma; ' $n$ annyways pit a pinch o' salt, a stra'aw, an' a button on the door sil o' nights, an' tha Horrors 'll no can coom ower it, light or no light."

So tha want ther wa'ays; an' as da'ays want by, an' niver a moon come, nat'rally tha talked-ma word! a reckon tha did ta'alk! ther tongues wagged like kenna what, at ho'am, an' at th' inn, an i' tha garth. But so come one da'ay, as tha sat on tha gra'at scttle i' th' Inn, a man fro' tha fa'ar en' $o$ ' th' boglan's was smokin' an listenin', when all to wanst, a sat oop 'n slapt's knec. "Ma faicks!" sa'ays 'e, "A 'd clean furgot, but a reckon a kens wheer tha moon be !" an' he tellt 'em hoo a wor lost i' tha bogs, an' hoo when a wor nigh de'ad wi' fright, tha loight shone oot, an' a' tha Evil Things fled awa'ay, an' a fund tha pad 'n got ho'am safe. 
"An' a wor so mazed wi' fear, loike," says he, "a didn't reetly look wheer the light comed fro'; but a mind fine 'twor saft an' white like tha moon's sel'. An 't comed fro' suthin' da'ark stannin' nigh a black snag i' tha watter. An' a didn't reetly look," says he age'an, "but a seem to mind a shinin' fa'ace an' yaller hair $i$ ' the mid'. $o$ ' the dazzle, an' 't'ad a sort o' kin' look, loike th' aud moon 'asel aboon tha Cars o' nights.

So aff tha a' want to tha wise woman, an' tellt un aboot it, an' a looked lang i' the pot an' tha Book age'an, an' than a nodded 's he'ad.

"Its da'ark still, childer, da'ark!" sayss she, "an' a canna reetly see owt, but do 's a tell ye, an' ye'll fin' out for yersel's. Go'a all on ye, just afwore the night gathers, pit a sto'on i' yer gobs, an' tak' a hazel twig i' yer han's, an' say ne'er a word till yer safe ho'am age'an. Than wa'alk on an' fear nowt, fair into tha mid' o' tha ma'ash, till ye fin' a coffin, a can'lle, an' a cross. Than ye 'll no be far frae yer moon ; look, and mappen ye 'll fin'.

Tha lookit each at ither, an' scratched the'r heads.

"But wheer 'll us fin' her, mother?" says ane.

"An' hoo 'll us goa?" says t'other.

"An wull na' tha bogles fett us?" says another, an' so on.

"Houts!" said she, fratched loike. "Passel o' fools! A can tell ye nae more; do as a tellt ce 'n fear nowt ; ' $n$ ' ef ye don't loike, than sta'ay by tha hoose, an' do wi' outen yer moon ef ye wull."

So cum tha nex' night i' tha darklin's, oot tha want a' thegether, ivery man wi' a sto'on in's moath, an' a hazeltwig in's han', an' feelin', thou mayst reckon, main feared an' creepy. An' tha stummelt an' stottered along tha pads into the mid o' tha bogs; tha seed nowt, mirover, thoff tha heerd sighin's an' flust'rin's i' ther ears, an' felt cowld wet fingers techin' 'em ; but on tha want, lookin' aroon' for tha coffin, tha can'le, an' tha cross, while tha comed nigh to the pool a side o' tha great snag, wheer the moon lay buried. An' a' towanst tha stopt, quakin' an' mazed an' skecry, fur theer wor tha gra'at sto'an, half in, half oot, o' tha watter, 
fur a' th' warl' loike a stra'ange big coffin ; an' at tha he'ad wor tha black snag, stretchin' oot's twae arms in a dark grewsome cross; an' on it a tiddy light flickered, like a deein' can'le. An' tha a' knelt down i' tha muck, an' crossed thersel's, an' said, "Our Lord", fu'st for'ard 'cause o' tha cross, an' then back'ard, to ke'p off tha Bogles; but wi'oot sp'akin' out, fur tha kenned as tha Evil Things 'd catch 'em, ef tha didna do as tha wise woman tellt 'em.

Than tha want nigher, an' tha took hol' on tha big sto'an, an' shoved un oop, an' arterwards tha said 'at fur wan tiddy minute, tha seed a stra'ange an' beautiful fa'ace lookin' oop at 'em glad loike oot o' tha black watter; but tha light coomed so quick 'an so white an' shinin', 'at tha stept ba'ack mazed wi' it, an' wi' tha gre'at angry wail as coomed fro' tha fleein' Horrors ; an' tha varry nex' minute, when they could see age'an, theer wor tha full moon $i$ ' tha sky, bright an' beautiful an' kin' 's 'iver, shinin' an' smilin' doun at 'em, an' makin' tha bogs an' tha pads as clear as da'ay, an' stealin' into tha varry corners, as thoff she'd ha' druv tha darkness an' tha Bogles clean awa'ay ef a could.

So ho'am tha Car-fo'ak want, gladly and wi' light hearts ; $a n$ ' iver sence tha moon shines brighter 'n clearer ower tha Bogs than ither wheers ; fur a mind's fine, 'at tha Horrors coom wi' tha da'ark, an' mischance an' mischief an' a' evil things, an' 'at tha Car-fo'ak sowt her an' found her, whan a wor de'ad an' buried i' tha Bog, an' ma'rk my wo'ds, it be a' true, fur ma gran 'asel a seed the snag wi' its twae arms, fur a' tha warl' loike a gre'at cross, an' tha green slimy watter at 's fut, wheer tha poor moon wor buried, an' the sto'an near by 'at kep' a doun, while tha wise woman sent's Car-fo'ak to set a loose, an' pit a in's sky age'an.

The following story is of a different character, more of what is known among folk-lorists as a Droll. It seems to be a continuation of the story Coat o' Clay, which I sent to $\mathrm{Mr}$. Lang some time ago, and which was printed by him in Longman's Magazine, and afterwards in FOLK-LORE. It was told me by the same person. 


\section{A Pottle o' Brains.}

Once i' these parts, an' not so long gone nayther, there was a fool as wanted to buy a pottle o' brains, for he was iver gettin' into scrapes through his foolishness, an' bein' laughed at by iveryonc. Fo'ak tellt him as he could get everything a liked from tha wise woman as lived on the top o' the hill, an' dealt in potions an' herbs an' spells an' things, an' could tell thee all as 'd come to thee or thy folk. So he tellt 's mother, 'n axed her if a should seek tha wise woman ' $n$ ' buy a pottle o' brains.

"That ye should," says she: "thou'st sore need o' them, my son; an' ef a should dee, who'd take care o' a poor fool such 's thou, no more fit to look arter thysel' than an unborn babby? but min' thy manners, an' speak her pretty, my lad; fur they wise fo'ak are gey'an light mispleased."

So off he went after 's tea, an' there she was, sittin' by tha fire, an' stirrin' a big pot.

"Good e'en, missis," says he, "its a fine night."

"Aye," says she, an' went on stirring.

"It'll mebbe rain," says he, an' fidgetted from one foot to t'other.

"Mlebbe," says she.

"An' mappen 't 'ull no," says he, an' looked out o' the window.

"Mappen," says she.

An' he scratched 's head, an' twisted 's hat.

"Wcel," says he, "a can't min' nuthin' else aboot tha weather, but lemme see; the crops is gittin' on fine."

"Fine," says she.

"An'-an'-tha beasts is fattenin'," says he.

"They are," says she.

"An'-an'-" say's he, 'n comes to a stop-"a reckon we'll tackle business noo, hevin' done tha perlite like. Hev' ye ony brains fur to sell?" 
"That depen's," says she, "ef thou wants king's brains, or sodger's brains, or schoolme'aster's brains, a dinina keep 'em."

"Hout no," says he, "jist ord'nar brains-fit fur any fool-same 's every one has 'bout here; suthin' clean common-like."

"Aye so," says tha wise woman, "a' might manage that, ef so be thou'll help thysel'."

"Hoo's that fur, missis?" says he.

"Jest so," says she, lookin' in 's pot; "bring me the heart o' tha thing thou likes best o' all, an' a 'll tell thee where to get thy pottle o' brains."

"But," says he, scratching his head, "hon can a do that?"

"That's no 'on fur me to say," says she, "fin' oot fur thysel', my lad! ef thou disna want to be a fool a' thy days. But thou 'll hev' to read me a riddle so 's a can see thou 'st brought the reet thing, an' ef thy brains is 'boot thee. An' a 've suthin' else to see to," says she, "so gode'en to 'ee," and she carried the pot away wi' her into tha back place.

So off goes the fool to 's mother, an' tellt her what tha wise woman said.

"An' a reckon a 'll hev to kill that pig," says he, "fur a like fat bacon better nor iverythin'."

"Then do 't, my lad," said 's mother, "fur sartain 't 'ull be a stra'ange an' good thing fur 'ee, ef thou canst buy a pottle $O$ ' brains, an' be able to look arter thy ain sel'."

So he killed 's pig, an' nex' day off a went to tha wise woman's cottage, an' there she sat, readin' in a great book.

"Gode'en, missis," say he, "a 've brought thee tha heart $o$ ' tha thing a likes best o' all; an' a put it hapt i' paper on tha table."

"Aye so?" says she, an' looked at him through her specitals. "Tell me this then, what rins wi'oot feet?" 


\section{Legends of the Cars.}

He scratched 's head, an' thowt, an' thowt, but a couldn't tell.

"Go thy ways," say's she, " thou'st no fo't me the reet thing yet. I'se no'on brains fur 'ee to-day". An' she clapt the book togither, an' t'orned 's back.

So off tha fool went to tell 's mother.

But as a got nigh the hoose, oot came fo'ak runnin' to tell un 'at 's mother was deein'.

$\mathrm{An}^{\prime}$ when he got in, 's mother ony looked at un, an' smiled, 's if to say she could leave un wi' a quiet min, sence a'd got brains 'nuff noo to look arter 's sel'-an' then she dee'd.

So doun a sat, an' the more a thowt aboot it the badder a feeled. He minded hoo she'd nuss't un when a wor a tiddy brat, an' he'ped un wi' 's lessons, an' cooked 's dinners, an' mended 's clouts, an' born wi' 's foolishness; an' a felt sorrier ' $n$ ' sorrier, while a began to sob an' greet.

"Oh, mother, mother!" says he, "who'll tak' care on me noo! Thou shouldn't hev' lef' me alo'an, fur a liked thee better nor iverything !"

An' as he said that, he thowt of the words o' the wise woman. " $\mathrm{Hi}$, yi !" says he, "must a cut oot mother's heart an' tak' it to her? A disna like the job," an' he took oot a knife an' felt 's edgc.

"No! a can't do 't," says he. "What'll a do ! what'll a do to get that pottle o' brains, noo a'm alone i' the worl'?" So a thowt an' thowt, an' next day a went an' borrowed a sack, an' bundelt 's mother in, an' carried it on 's showther up to th' wise woman's cottage.

"Gode'en, missis," says he, "a reckon a 've fo't 'ee the reet thing this time, surely," an' he plumped the sack down kerflap! in the doorsil.

"Mebbe," says the wise woman, "but read me this, noo, what's yaller an' shinin' but isna goold ?"

An' he scratched 's head, an' thowt, an' thowt, but a couldna tell.

"Thou'st no hit the reet thing, my lad," says she. "I 
doubt thou's a bigger fool nor a thought!" an' shut the door in 's face.

"See there!" says he, an' sets doun by tha road side an' greets.

"A've lost tha on'y twae things as a cared for, an' what else can a fin' to buy a pottle o' brains wi' !" an' he fair howled, till tha tears ran doun into 's mooth. An' oop came a lass as lived gainhand, an' looked at un.

"What's oop wi' thee, fool?" says she.

"Oo a's killed ma pig, 'n lost my mother, an' a'm nobbut a fool mysel'," says he, sobbin'.

"That's bad," says she; "an' hevna thee anybody to look arter thee?"

"Naw," says he, "an' a canna buy my pottle o' brains fur thurs nuthin' a like best lef'!"

"What art ta'alkin' aboot"! says she.

An' doun she sets by him, an' he tellt her all aboot the wise woman an' the pig, an' 's mother an' the riddles, an' 'at he was alo'an i' the warld.

"Weel," says she, "a wouldn't min' lookin' arter thee mysel?"."

"Could thee do ' $t$ ?" says he.

"Ou, ay!" says she, "fo'ak says as fools mak' good husban's, an' a reckon a'll hev thee, ef thou'st willin'."

"Can'st cook ?" says he.

"Ay, a can," says she.

"An' scrub ?" says he.

"Surely," says she.

"An' men' ma clouts ?" says he.

"A can that," says she.

"A reckon thou'lt do then 's weel 's anybody," says he; "but what 'll a do 'bout this wise woman ?"

"Oh, wait a bit," says she, "suthin' mowt turn up, an' it 'll no matter ef thou 'rt a fool, s' long 's thou'st got me to look arter thee."

"That's true," says he, an' off tha went and got married. 
An' she kept 's house so clean an' neat, an' cooked 's dinner so fine, 'at one night a says to her :

"Lass, a 'm thinkin' a like thee best o' iverything, arter all."

"That's good hearin'," says she, "an' what then ?"

"Hev 'a got to kill thee, dost think, an' take thy heart oop to the wise woman for that pottle o' brains?"

"Laws, no!" says she, lookin' skeered, "a winna hev' that. But see here; thou didn't cut oot thy mother's heart, did tha?"

"Naw; but if a had, mebbe a'd a got my pottle o' brains," says he.

"Not a bit o't," says she; "jist thou take me 's a be, heart 'n all, 'n a wager a 'il help thee read the riddles."

"Can thee so?" says he, doubtful like: "a reckon thon 's too hard for wimmen fo'ak."

"Weel," says she, "let 's see noo. Tell 's the first 'un."

"What rins wi' oot feet ?" says he.

"Why, watter !" says she.

"It do," says he, an' scratched 's head.

"An' what 's yaller an' shinin', but isna goold ?"

"Why, the sun!" says she.

"Faix, it be !" says he. "Coom, us 'll go oop to the wise woman towanst," and off they went. $A n^{\prime}$ as they comed oop the pad, she wor sittin' at the door, twinin' straws.

"Gode'en, missis," says he.

"Gode'en, fool," says she.

"A reckon a 's fo't 'e the reet thing to last," says he, "thoff a hern't azac'ly cut th' heart oot, it be so moocky wark."

The wise woman looked at 'em both, an' wiped her spec'itals.

"Canst tell me what that be, as has first nae legs, an' then twae lcgs, an' en's wi' fower legs?"

An' the fool scratched 's head, an' thowt, an' thowt ; but a couldna tell. 
An' the lass whispered in 's ear :

"It be a tadpole."

"Mappen," says he then, "it mout be a tadpole, missis." The wise woman nodded 's head.

"That 's reet," says she, "an' thou'st got thy pottle o' brains a'ready."

"Wheer be they ?" says he, lookin' aboot, an' feelin' in 's pockets.

"In thy wife's head," says she. "The on'y cure fur a fool 's a good wife to look arter 'n, an' that thou'st got; so gode'en to 'ee!" An' wi' that she nodded to 'em, an' up and into the hoose.

So they went ho'am together, an' a niver wanted to buy a pottle o' brains age'an, fur's wife 'ad enuff fur both.

M. C. BALFOUR. 